\title{
ÜBER DIE ENTWICKLUNG COMPLEXER GRÖSSEN
}

\section{IN KETTENBR ÜCHE}

\author{
VON
}

\section{A. HURWITZ}

in $\mathrm{KONIGSBERG} \mathrm{i} / \mathrm{Pr}$.

Es möge $(S)$ ein System von Zahlen bezeichnen, welches die Eigenschaft besitzt, dass die Summe, die Differenz und das Produkt irgend zweier Zahlen des Systems wieder Zahlen des Systems sind. ${ }^{1}$ Wenn die complexen Grössen in der üblichen Weise durch die Punkte einer Ebene dargestellt werden, so wird den Zahlen von $(S)$ ein gewisses System von Punkten entsprechen. Ich nehme an, das System $(S)$ sei so beschaffen, dass von diesen Punkten in jedem endlichen Gebiete der Ebene nur eine endliche Anzahl liegt. Daraus folgt, dass ausser der Null keine andere Zahl von $(S)$ existirt, deren absoluter Betrag kleiner als I ist. Denn die Potenzen dieser Zahl würden sämmtlich Zahlen von $(S)$ sein und im Innern des um den Nullpunkt mit dem Radius I beschriebenen Kreises liegen. Eine letzte Voraussetzung, die ich in Betreff des Systems $(S)$ mache, ist die, dass die Zahl I dem Systeme angehört.

Von einer Grösse $x_{0}$ ausgehend bilde ich nun die Gleichungskette:

$$
\begin{gathered}
x_{0}=a_{0}+\frac{\mathrm{I}}{x_{1}}, \quad x_{1}=a_{1}+\frac{\mathrm{I}}{x_{2}}, \quad x_{2}=a_{2}+\frac{\mathrm{I}}{x}, \ldots \\
x_{n}=a_{n}+\frac{\mathrm{I}}{x_{n+1}}, \ldots
\end{gathered}
$$

1 Eine Theorie solcher Zahlsysteme ist in den bekannten Arbeiten von Kronecker und DeDEKIND, vorzugsweise für den Fall algebraischer Zahlen, entwickelt. Vgl. insbesondere das XI. Supplement zu Dirichler's Vorlesungen über Zahlentheorie. Dritte Auflage. Acta mathematica. 11. Imprimé le 6 Mars 1888. 
wo $a_{0}, a_{1}, a_{2}, \ldots, a_{n}, \ldots$ irgend welche Zahlen des Systems $(S)$ bedeuten. Ich nehme an, dass sich die Gleichungskette (I) in's Unendliche fortsetzt, und dass alle auftretenden Grössen $x_{1}, x_{2}, \ldots$ sowie die Zahlen $a_{0}, a_{1}, \ldots$ endliche Werthe besitzen. Die Elimination von $x_{1}, x_{2}, \ldots, x_{11}$ aus den ersten $n+$ I Gleichungen (I) ergiebt die Darstellung von $x_{0}$ in Form eines Kettenbruches, welchen ich mit

$$
x_{0}=\left(a_{0}, a_{1}, a_{2}, \ldots, a_{n}, x_{n+1}\right)
$$

bezeichnen will und über welchen ich folgende Voraussetzungen mache:

Wenn der $n^{\text {te }}$ Näherungsbruch mit

$$
\frac{p_{n}}{q_{n}}=\left(a_{0}, a_{1}, a_{2}, \ldots, a_{n}\right)
$$

bezeichnet, und

$$
x_{0}-\frac{p_{n}}{q_{n}}=\frac{\theta_{n}}{q_{n}^{3}}
$$

gesetzt wird, so sei der absolute Betrag von $\theta_{n}$ für alle Werthe von $n$ kleiner als eine endliche Grösse $\rho$, dagegen wachse der absolute Betrag von $q_{n}$ mit zunehmendem $n$ über alle Grenzen.

Falls alle diese Voraussetzungen zutreffen, lässt sich Folgendes erschliessen:

Erstens; Der in's Unendliche fortgesetzte Kettenbruch

$$
\left(a_{0}, a_{1}, a_{2}, a_{3}, \ldots\right)
$$

convergirt und sein Werth ist $x_{0}$. Denn der Gleichung (4) zufolge wird die Differenz $x_{0}-\frac{p_{n}}{q_{n}}$ unendlich klein, wenn $n$ uber alle Grenzen wächst.

Zweitens; Die Grösse $x_{0}$ kann nicht gleich dem Quotienten zweier Zahlen $r, s$ des Systems $(S)$ sein. Angenommen nämlich es sei $x_{0}=\frac{r}{s}$, so folgt

$$
r q_{n}-s p_{n}=\frac{s \cdot \theta_{n}}{q_{n}}
$$

Mit wachsendem $n$ wird daher $r q_{n}-s p_{n}$ dem absoluten Betrage nach 
unendlich klein. Wählt man nun $n$ so gross, dass dieser absolute Betrag kleiner ist als $\mathbf{I}$, so muss

$$
r q_{n}-s p_{n}=0
$$

sein, weil o die einzige Zahl des Systems $(S)$ ist, deren absoluter Betrag kleiner ist als I. Es ist also

$$
x_{0}=\frac{r}{s}=\frac{p_{n}}{q_{n}}=\left(a_{0}, a_{1}, \ldots, a_{n}\right) .
$$

Der Vergleich dieser Gleichung mit (2) ergiebt $x_{n+1}=\infty$, was der Annahrne alle Grössen $x_{1}, x_{2}, \ldots$ seien endlich widerstreitet.

Drittens: Genügt die Grösse $x_{0}$ einer quadratischen Gleichung, deren Coefficienten Zahlen des Systems $(S)$ sind, so kommen in der unendlichen Reihe

$$
x_{1}, x_{2}, x_{3}, \ldots, x_{n}, \ldots
$$

nur eine endliche Anzahl verschiedener Grössen vor.

Ich entwickle, um den Beweis dieses Satzes zu führen, zunächst einige Hülfsformeln. Bekanntlich ist

$$
x_{0}=\frac{p_{n} x_{n+1}+p_{n-1}}{q_{n} x_{n+1}+q_{n-1}}
$$

woraus, in Rücksicht auf die Relation

$$
p_{n} q_{n-1}-q_{n} p_{n-1}=(-\mathrm{I})^{n-1},
$$

folgt:

$$
\left\{\begin{array}{c}
x_{0}-\frac{p_{n}}{q_{n}}=\frac{(-1)^{n}}{q_{n}^{2}\left(x_{n+1}+\frac{q_{n-1}}{q_{n}}\right)}, \\
x_{0}-\frac{p_{n-1}}{q_{n-1}}=\frac{(-1)^{n-1}}{q_{n-1}^{2}\left(\frac{1}{x_{n+1}}+\frac{q_{n}}{q_{n-1}}\right)} .
\end{array}\right.
$$

Vergleicht man diese Formeln mit der Gleichung (4), so ergiebt sich

$$
\left\{\begin{array}{l}
x_{n+1}+\frac{q_{n-1}}{q_{n}}=\frac{(-1)^{n}}{\theta_{n}}, \\
\frac{1}{x_{n+1}}+\frac{q_{n}}{q_{n-1}}=\frac{(-1)^{n-1}}{\theta_{n-1}} .
\end{array}\right.
$$


Daher ist der absolute Betrag sowohl von

$$
x_{n+1}+\frac{q_{n-1}}{q_{n}}, \text { wie von } \frac{\mathrm{I}}{x_{n+1}}+\frac{q_{n}}{q_{n-1}}
$$

stets grǒsser als $\rho^{\prime}=\frac{1}{\rho}$, wo die Grösse $\rho^{\prime}$ von Null verschieden ist. Sei nun

$$
A x^{2}+B x+C=\text { o }
$$

die Gleichung, welcher $x_{0}$ genügt; sei ferner

$$
B^{2}-4 A C=D \text {. }
$$

Dann ist $x_{n+1}$, der Gleichung (5) zufolge, Wurzel der Gleichung

$$
A\left(p_{n} x^{\prime}+p_{n-1}\right)^{2}+B\left(p_{n} x^{\prime}+p_{n-1}\right)\left(q_{n} x^{\prime}+q_{n-1}\right)+C\left(q_{n} x^{\prime}+q_{n-1}\right)^{2}=0
$$

welche in geordneter Form

$$
A^{\prime} x^{\prime 2}+B^{\prime} x^{\prime}+C^{\prime}=0
$$

lauten möge. Hier bezeichnen $A^{\prime}, B^{\prime}, C^{\prime}$ Zahlen des Systems $(S)$, und es ist

$$
B^{2}-4 A^{\prime} C^{\prime}=D
$$

Bezeichnet nun $y_{n+1}$ die zweite Wurzel von (II), so ist die Grösse

$$
y_{0}=\frac{p_{n} y_{n+1}+p_{n-1}}{q_{n} y_{n+1}+q_{n-1}}
$$

die zweite Wurzel der Gleichung (9). Diese zweite Wurzel $y_{0}$ ist von der ersten Wurzel $x_{0}$ verschieden, weil andernfalls $y_{0}=x_{0}=\frac{-B}{2 A} \mathrm{der}$ Quotient zweier Zahlen des Systems $(S)$ sein würde, was, wie oben gezeigt wurde, nicht sein kann. Aus (13) folgt:

$$
y_{n+1}=\frac{q_{n-1} y_{0}-p_{n-1}}{--q_{n} y+p_{n}}=-\frac{q_{n-1}}{q_{n}}+\frac{(-1)^{n-1}}{-q_{n}^{2} y_{0}+p_{n} q_{n}},
$$


Über die Entwicklung complexer Grössen in Kettenbrüche.

oder, wenn $p_{n}$ mit Hülfe von (4) eliminirt wird: ${ }^{1}$

$$
y_{n+1}=-\frac{q_{n-1}}{q_{n}}+\frac{(-1)^{n-1}}{q_{n}^{2}\left(x_{0}-y_{0}\right)-\theta_{n}} .
$$

Da nun $x_{0}-y_{0}$ eine von Null verschiedene Grösse ist, die von $n$ unabhängig ist, so werden in den Gleichungen

$$
y_{n+1}=-\frac{q_{n-1}}{q_{n}}+\varepsilon_{n}, \quad \frac{1}{y_{n+1}}=-\frac{q_{n}}{q_{n-1}}+\varepsilon_{n}^{\prime}
$$

die Grössen $\varepsilon_{n}$ und $\varepsilon_{n}^{\prime}$ mit wachsenden Werthen von $n$ unendlich klein. Die rechten Seiten der Gleichungen

$$
\begin{gathered}
x_{n+1}-y_{n+1}=\left(x_{n+1}+\frac{q_{n-1}}{q_{n}}\right)-\varepsilon_{n} \\
\frac{\mathrm{I}}{x_{n+1}}-\frac{\mathrm{I}}{y_{n+1}}=\left(\frac{\mathrm{I}}{x_{n+1}}+\frac{q_{n}}{q_{n-1}}\right)-\varepsilon_{n}^{\prime}
\end{gathered}
$$

sind daher, wenn $n$ eine bestimmte Grenze überschreitet, den absoluten Betrage nach grösser als $\rho^{\prime \prime}$, wo $\rho^{\prime \prime}$ eine um beliebig wenig kleiner als $\rho^{\prime}$ angenommene Grösse bedeutet.

Durch Auflösung der Gleichung (I I) findet man aber

$$
x_{n+\mathrm{r}}-y_{n+1}=\frac{ \pm \sqrt{D}}{A^{\prime}}, \quad \frac{\mathrm{I}}{x_{n+1}}-\frac{\mathrm{I}}{y_{n+1}}=\frac{\mp \sqrt{D}}{C^{\prime}},
$$

und also ist, von einem bestimmten Werthe von $n$ ab,

$$
\left|A^{\prime}\right|<\frac{|\sqrt{D}|}{\rho^{\prime \prime}}, \quad\left|C^{\prime}\right|<\frac{|\sqrt{D}|}{\rho^{\prime \prime}} .
$$

Folglich können $A^{\prime}, C^{\prime}$ und $B^{\prime}=\sqrt{D+4 A^{\prime} O^{\prime}}$, sowie

$$
x_{n+1}=\frac{-B^{\prime} \pm}{2 A^{\prime}} \frac{\sqrt{D}}{}
$$

${ }^{1}$ Für den Fall der Entwicklung reeller Grössen in Kettenbrüche, deren Theilnenner gewöhnliche reelle positive ganze Zablen sind, hat Herr HERMre diese Gleichung zum Beweise der periodischen Entwicklung quadratischer Irrationalitäten verwendet. (Bulletin des sciences mathématiques, $2^{\text {me }}$ série, t. 9, pag. II.) 
von diesem Werthe von $n$ ab, nur noch eine endliche Anzahl verschiedener Werthe annehmen. Es sind also in der That, wie behauptet wurde, in der Reihe

$$
x_{1}, x_{2}, x_{3}, \ldots
$$

nur eine endliche Anzahl verschiedener Grössen vorhanden.

Indem ich mich nunmehr den Anwendungen der entwickelten Sätze auf besondere Zahlensysteme $(S)$ zuwende, bemerke ich vorab noch Folgendes: Die Voraussetzungen (V) sind sicher erfüllt, wenn der absolute Betrag von $\frac{q_{n}}{q_{n-1}}$ beständig grösser als I und der absolute Betrag von $\frac{I}{x_{n}}$ beständig kleiner als $k$ ist, wo $k$ eine positive Zahl kleiner als I bezeichnet. In der That wăchst dann der absolute Betrag von $q_{n}$, der Ungleichung $\left|q_{n}\right|>\left|q_{n-1}\right|$ zufolge, mit $n$ über alle Grenzen, und es ist wegen (8),

$$
\left|\frac{\mathrm{I}}{\theta_{n-1}}\right| \geq\left|\frac{q_{n}}{q_{n-1}}\right|-\left|\frac{\mathrm{I}}{x_{n+1}}\right|>\mathrm{I}-k,
$$

also der absolute Betrag von $\theta_{n}$ beståndig kleiner als $\frac{\mathrm{I}}{\mathrm{I}-k}$.

I.

(S) ist das System der complexen ganzen Zahlen $m+n i$.

Es sei $x=u+i v$ eine beliebige complexe Grösse. Ich setze

$$
x=a+\left(u^{\prime}+i v^{\prime}\right),
$$

wo die complexe ganze Zahl $a$ so bestimmt werden soll, dass

$$
-\frac{1}{2} \leqq u^{\prime}<\frac{1}{2} \text { und }-\frac{1}{2} \leqq v^{\prime}<\frac{1}{2}
$$

wird. Auf diese Weise wird jeder complexen Grösse $x$ eine bestimmte complexe ganze Zahl a zugeordnet. Bildet man nun, von irgend einer Grösse $x_{0}$ ausgehend, die Gleichungskette

$$
\text { (16) } \quad x_{0}=a_{0}+\frac{1}{x_{1}}, \quad x_{1}=a_{1}+\frac{1}{x_{2}}, \ldots, x_{n}=a_{n}+\frac{1}{x_{n+1}}, \ldots
$$


wobei allgemein $a_{n}$ diejenige complexe ganze Zahl bezeichnet, welche der Grösse $x_{n}$ zugeordnet ist, so ist zunächst

$$
\left|\frac{\mathrm{I}}{x_{n}}\right| \leqq \sqrt{\frac{\mathrm{I}}{2}}
$$

Der Beweis der Ungleichung $\left|q_{n}\right|>\left|q_{n-1}\right|$ ist nicht ganz einfach; er erfordert eine genauere Untersuchung der Zahlenreihe $a_{1}, a_{2}, \ldots$ Behufs dieser Untersuchung zerlege ich die Zahlenebene durch die Geraden

$$
u= \pm \frac{\mathrm{r}}{2}, \pm \frac{3}{2}, \pm \frac{5}{2}, \ldots ; \quad v= \pm \frac{\mathrm{I}}{2}, \pm \frac{3}{2}, \pm \frac{5}{2}, \ldots
$$

in unendlich viele Quadrate. Dann wird jeder Grösse $u+i v$ diejenige complexe ganze Zahl zugeordnet sein, welche den Mittelpunkt des die

Fig. I.

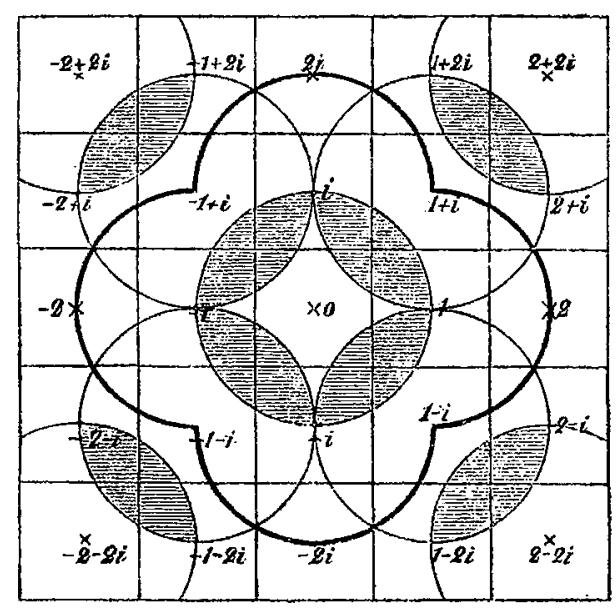

Grösse $u+i v$ enthaltenden Quadrates bildet, wobei von den Rändern des einzelnen Quadrates nur diejenigen zu dem Quadrate rechnen, welche man vom Mittelpunkte aus nach der Richtung der abnehmenden $u$, bez. $v$ erblickt. Da $x_{0}-a_{0}$ in dem Quadrate mit dem Mittelpunkte o liegt, so wird $x_{1}=\frac{1}{x_{0}-a_{0}}$ dem Raume $R$ angehören, welcher ausserhalb der Kreise (I), $(i),(-\mathrm{I}),(-i)$ liegt. ${ }^{1}$ Hier habe ich, wie in der Folge stets, mit $(a)$ denjenigen Kreis bezeichnet, welcher den Radius I und den

${ }^{1}$ Die Begrenzung von $R$ ist in Figur I. scharf gezeichnet.

Acta mathematica. 11. Imprimé te 6 Mars 1888. 
Punkt a zum Mittelpunkt hat. Wie $x_{1}$ werden auch $x_{2}, x_{a}, \ldots$ in den Raum $R$ fallen, und daher können die Zahlen $a_{2}, a_{2}, a_{3}, \ldots$ keinen der Werthe $o,+\mathbf{I},+i,-\mathrm{I},-i$, annehmen. Aber die Zahlenreihe $a_{1}$, $a_{2}, a_{3}, \ldots$ unterliegt ferner noch gewissen Beschrănkungen, welche sich auf die Aufeinanderfolge der Zahlen beziehen. Sei beispielsweise

$$
a_{n}=2+i \text {. }
$$

Dann muss $x_{n}$ sowohl dem P,aume $R$ als auch dern Quadrate mit dem Mittelpunkte $2+i$ angehören. Folglich kann $x_{n+1}=\frac{1}{x_{n}-a_{n}}$ nicht in denjenigen Raum eintreten, welcher aus $R$ von dem Kreise $(-\mathrm{I}+i)$ ausgeschnitten wird. Daher ist die Folge $a_{n}=2+i, a_{n+1}=-1+i$ unmöglich. Man erkennt sofort, dass solche Beschränkungen in der Aufeinanderfolge der Zahlen $a$ immer und nur dann bei der Zahl $a_{n}$ beginnen werden, wenn das Quadrat mit dem Mittelpunkte $a_{n}$ von der Begrenzung des Raumes $R$ durchschnitten wird. Fur den vorliegenden Zweck genügt es von den Zahlfolgen $a_{n}, a_{k+1}, \ldots$ die folgenden als unmöglich zu constatiren, was an der Hand von Fig. I. ohne Schwierigkeit geschieht.

Tabelle unmöglicher Zahlfolgen.

\begin{tabular}{|c|c|c|c|c|}
\hline & $a_{n}$ & $a_{n+1}$ & $a_{n+2}$ & $a_{n+3}$ \\
\hline I. & $-2,2 i,-1+i,-2+i,-1+2 i$ & $1+i$ & & \\
\hline II. & $2,2 i, \mathbf{1}+i$ & $-2+2 i$ & & \\
\hline III. & $2+i, 1+2 i$ & $-2+2 i$ & $1+i$ & \\
\hline IV. & $-2,2 i,-\mathrm{I}+i$ & $2+2 i$ & & \\
\hline $\mathrm{V}$. & $-2+i,-1+2 i$ & $2+2 i$ & $-2+2 i$ & $1+i$ \\
\hline
\end{tabular}

Wenn ferner $a_{n}=2+i$ oder $\mathrm{I}+2 i$ ist, und die folgenden Zahlen $a_{n+1}, a_{n+2}, \ldots, a_{n+2 k-1}$ haben abwechselnd die Werthe $-2+2 i$ und $2+2 i$, so kann $a_{n+2 k}$ nicht gleich $I+i$ sein; ebenso wenn $a_{n}=-2+i$ oder $-1+2 i$ ist und die folgenden Zahlen $a_{n+1}, a_{n+2}, \ldots, a_{n+2 k}$ haben abwechselnd die Werthe $2+2 i$ und $-2+2 i$, so kann $a_{n+2 k+1}$ nicht gleich $1+i$ sein. Die einfachsten Falle hierfür sind in der Tabeile unter III. und V. aufgenommen. 
Dies vorausgeschickt gehe ich nun zum Beweise der Ungleichung $\left|q_{n}\right|>\left|q_{n-1}\right|$ aber. Ich setze

$$
k_{n}=\frac{q_{n}}{q_{n-1}}
$$

dann ist, der Gleichung $q_{n}=a_{n} q_{n-1}+q_{n-2}$ zufolge:

$$
k_{1}=a_{1}, \quad k_{2}=a_{2}+\frac{\mathrm{I}}{k_{1}}, \ldots, \quad k_{n}=a_{n}+\frac{\mathrm{I}}{k_{n-1}}, \ldots,
$$

und es ist zu beweisen, dass beständig

$$
\left|k_{n}\right|>\mathbf{I}
$$

ist, oder dass der Punkt $k_{n}$ bestandig ausserhalb des Kreises (o) liegt. Dies trifft nun für $k_{1}$ offenbar $\mathrm{zu}$; ich will annehmen, dass $k_{1}, k_{2}, \ldots, k_{n-1}$ der Ungleichung (20) genügen, dagegen $k_{n}$ nicht mehr und werde zeigen, dass diese Annahme auf einen Widerspruch führt. Da $k_{n}=a_{n}+\frac{\mathrm{I}}{k_{n-1}}$ im Innern des Kreises $\left(a_{n}\right)$ liegt, so muss $a_{n}$ einen der Werthe $\mathrm{I}+i$, $\mathrm{I}-i,-\mathrm{I}+i,-\mathrm{I}-i$ besitzen; denn in allen anderen Făllen würde $k_{n}$ ausserhalb des Kreises (o) fallen, also der absolute Betrag von $k_{u}$ grösser als $\mathrm{I}$ sein. Ich betrachte nun nur den Fall $a_{n}=\mathrm{I}+i$, da die drei übrigen Fälle eine ganz analoge Behandlung gestatten.

Da $k_{n}=a_{n}+\frac{\mathrm{I}}{k_{n-1}} \mathrm{im}$ Innern der beiden Kreise $(\mathrm{o})^{\prime}$ und $(\mathbf{I}+i)$ liegt (den Rand des ersteren Kreises eingeschlossen, was durch das an (o) gesetzte Komma angedeutet werde) so fällt $\frac{\mathrm{I}}{r_{n-1}}$ in das Innere der Kreise (o) und $(-\mathrm{I}-i)^{\prime} ;^{1}$ folglich $k_{n-1}=a_{n-1}+\frac{\mathrm{I}}{k_{n-2}}$ in das Äussere des Kreises (o) und in das Innere von $(-\mathrm{I}+i)^{\prime}$. Daher kann $a_{n-1}$ nur einen der Werthe $-2,-2+i,-\mathrm{I}+2 i, 2 i,-\mathrm{I}+i,-2+2 i$ besitzen; denn in allen übrigen Fällen wird der Kreis $\left(a_{n-1}\right)$, welcher $a_{n-1}+\frac{\mathrm{I}}{k_{n-2}}$ in sich aufnimmt, nicht in das Innere des Kreises $(-\mathrm{I}+i)^{\prime}$ eintreten. Von jenen Werthen ist aber, der aufgestellten Tabelle zufolge, nur der letzte zulässig. Es muss also $a_{n-1}=-2+2 i$ sein. Da nun $a_{n-1}+\frac{1}{k_{n-2}}$

1 Die betreffenden Gebiete sind in der Figur 1. schraffirt. 
im Innern der beiden Kreise $(-2+2 i)$ und $(-\mathrm{I}+i)^{\prime 1}$ liegt, so folgt weiter, dass $k_{n-2}=a_{n-2}+\frac{\mathrm{I}}{k_{n-3}}$ ausserhalb des Kreises (o) und im Innern des Kreises $(\mathrm{I}+i)^{\prime}$ liegt, und hieraus, wieder mit Rücksicht auf die Tabelle, $a_{n-2}=2+2 i$. So fortfahrend erkennt man, dass die Zahlen $a_{n}, a_{n-1}, \ldots$ die Werthe haben müssen:

$$
\begin{gathered}
a_{n}=\mathrm{I}+i, \quad a_{n-1}=-2+2 i, \quad a_{n-2}=2+2 i \\
a_{n-3}=-2+2 i, \quad a_{n-4}=2+2 i, \ldots \ldots
\end{gathered}
$$

Bildet man aber mit diesen Zahlen die Grössen $k_{1}, k_{2}, k_{3}, \ldots, k_{n-1}$, so zeigt sich, dass sie sämmtlich in den unendlichen, von den Kreisen $(\mathrm{I}+i),(\mathrm{I}-i),(-\mathrm{I}+i),(-\mathrm{I}-i)$ begrenzten Raum fallen. Daher wird $\left|k_{n}\right|>$ I sein, was der Annahme widerstreitet. In ganz entsprechender Weise ergiebt sich ein Widerspruch, wenn man voraussetzt $a_{n}$ habe einen der Werthe $\mathrm{r}-i,-\mathrm{I}+i,-\mathrm{I}-i$.

Hiermit ist ausser Zweifel gesetzt, dass $\left|k_{n}\right|$ beständig grösser als I, also stets

$$
\left|q_{n}\right|>\left|q_{n-1}\right|
$$

ist. Auf Grund der vorausgeschickten allgemeinen Entwicklungen kann man nunmehr offenbar den folgenden Satz aussprechen:

Man entwickle eine beliebige complexe Grösse $x_{0}$ in einen Kettenbruch, indem man

$$
x_{0}=a_{0}+\frac{1}{x_{1}}, \quad x_{1}=a_{1}+\frac{1}{x_{3}}, \quad x_{2}=a_{2}+\frac{1}{x_{3}}, \ldots
$$

setzt, wo die ganze complexe Zahl $a_{n}$ immer so bestimmt ist, dass sowohl der reelle Bestandtheil, wie auch der Factor von $i$ in der Differenz $x_{n}-a_{n}$ zwischen $-\frac{\mathrm{I}}{2}$ und $+\frac{\mathrm{I}}{2}$ liegt. Dann wird dieser Kettenbruch $\mathrm{I}^{\circ}$ stets gegen den Werth $x_{0}$ convergiren, $2^{\circ}$ immer und nur dann abbrechen, wenn $x_{0}$ eine complexe rationale $Z$ Zahl ist, und $3^{\circ}$ stets periodisch werden, wenn $x_{0}$ einer Gleichung zweiten Grades mit ganzzahligen complexen Coefficienten genügt, ohne Wurzel einer eben solchen Gleichung ersten Grades zu sein. 
Ich brauche kaum besonders hervorzuheben, dass auf diesen Satz die Theorie der quadratischen Formen im Gebiete der complexen ganzen Zahlen gegründet werden kann, dass insbesondere aus ihm ohne Weiteres die Lösung der PeLL'schen Gleichung

$$
t^{2}-D u^{2}=I
$$

folgt, unter $D$ eine gegebene nichtquadratische, unter $t$ und $u$ zu bestimmende complexe ganze Zahlen verstanden. ${ }^{1}$

Ausser der hier betrachteten giebt es übrigens noch andere Kettenbruchentwicklungen im Gebiete der complexen ganzen Zahlen $m+n i$, für welche der obige Satz ebenfalls gilt, worauf ich indessen an dieser Stelle nicht eingehen will.

II.

(S) ist das System der complexen yanzen Zahlen $m+n \rho$

$$
\left(\rho=\frac{-1+\sqrt{-3}}{2}\right) .^{2}
$$

Ich verbinde den Punkt o mit den umliegenden Punkten I, I $+\boldsymbol{\rho}$, $\rho,-\mathrm{I},-\mathrm{I}-\rho,-\rho$ und crrichte in den Mitten der Verbindungslinien die Lothe. Diese bilden ein reguläres Sechseck mit dem Mittelpunkte o. (Vgl. Fig. 2.) Von den Randpunkten rechne ich nur die links von der Axe der rein imaginären Zahlen liegenden zu dem Sechseck. Wird in entsprechender Weise um jeden Punkt $m+n \rho$ ein solches Sechseck construirt, so uberdeckt die Gesammtheit der letzteren die complexe Zahlenebene einfach und lückenlos. Einer beliebigen complexen Grösse $x$ ordne ich nun diejenige ganze Zahl $m+n \rho$ zu, welche den

1 Vgl. die Andeutung in DrRicuners A Abhandiung: Recherches sur les formes quadratiques à coefficients et $\grave{a}$ indéterminées complexes. CRELL's Journal, Bd. 24, pag. 336.

2 Der Euclidische Algorithmus, für die Zahlen $m+n \rho$, welchen Herr Bachmans in seinem Buche: die Lehre von der Kreistheilung pag. 189 benutzt, ergiebt eine andere Kettenbruchentwicklung wie die, welohe ich im Texte definire. Die erstere Entwicklung legt eine Eintheilung der Ebene in Rechtecke zu Grunde, deren Mittelpunkte die ganzen Zahlen $m+n \rho$ sind, deren Seiten den Axen der reellen bez, rein imaginären Zahlen parallel laufen und bez. die Längen $I$ und $\frac{1}{2} \sqrt{3}$ besitzen. 
Mittelpunkt des die Grösse $x$ aufnehmenden Sechsecks bildet. Um eine beliebige Grösse $x_{0}$ in einen Kettenbruch $z u$ entwickeln setze ich

$$
x_{0}=a_{0}+\frac{1}{x_{1}}, \quad x_{1}=a_{1}+\frac{\mathrm{I}}{x_{2}}, \quad x_{2}=a_{2}+\frac{\mathrm{I}}{x_{3}}, \ldots,
$$

wobei allgemein $a_{n}$ die der Grösse $x_{n}$ zugeordnete Zahl bezeichnet. Da $\frac{1}{x_{n}}$ in das um den Nullpunkt abgegrenzte Sechseck fällt, so gilt die Un. gleichung

$$
\left|\frac{1}{x_{n}}\right| \leq \sqrt{\frac{1}{3}}
$$

$(n=1,2,3, \ldots)$

Um zu beweisen, dass der absolute Betrag von $k_{n}=\frac{q_{n}}{q_{n-1}}$ stets grösser ist als 1, bemerke ich zunächst, dass $x_{n}=\frac{1}{x_{n-1}-a_{n-1}}$ in den ausserhalb der Kreise $(\mathrm{I}),(\mathrm{I}+\rho),(\rho),(-\mathrm{I}),(-\mathrm{I}-\rho),(-\rho)$ liegenden Raum $R$ fällt. ${ }^{1}$ Hieraus folgt, dass die Zahlen $a_{1}, a_{2}, a_{3}, \ldots$ keinen der Werthe

Fig. 2

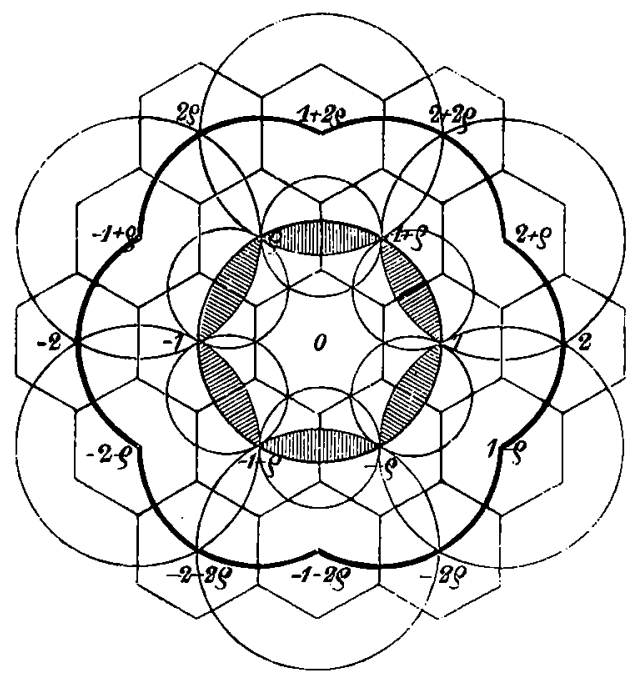

$\circ, \mathrm{I}, \mathrm{I}+\rho, \rho,-\mathrm{I},-\mathrm{I}-\rho,-\rho$ annehmen können. Die Begrenzung des Raumes $R$ durchsetzt, wie aus der Figur 2. ersichtlich ist, zwölf

\footnotetext{
${ }^{1}$ Die Begrenzung von $R$ ist in Fig. 2. scharf gezeichnet.
} 
Sechsecke. Daher giebt es wieder bestimmte Reihenfolgen der Zahlen $a_{n}, a_{n+1}, a_{n+2}, \ldots$, welche in der Gleichungskette (2x) nicht vorkornmen können. Für den vorliegenden Zweck genügt es zu bemerken, dass die Zahl $a_{n+1}$ nicht den Werth $2+\rho$ erhalten kann, wenn $a_{n}$ einen der Werthe $-2, \rho-1,2 \rho$ besitzt. Angenommen nun in der Reihe der Grössen

$$
k_{1}=a_{1}+\frac{1}{k_{0}}, \quad k_{2}=a_{2}+\frac{1}{k_{\mathrm{i}}}, \ldots, \quad k_{n}=a_{n}+\frac{1}{k_{n-1}}, \ldots,
$$

(wo $k_{0}=\infty, k_{1}=a_{1}$ ) sei $k_{n}$ die erste deren absoluter Betrag nicht grösser ist als I. Da $a_{n}+\frac{\mathrm{I}}{k_{n-1}}$ im Innern des Kreises $\left(a_{n}\right)$ liegt, so kann $a_{n}$ nur einen der Werthe

$$
2+\rho, \mathrm{I}+2 \rho, \rho-\mathrm{I},-2-\rho,-\mathrm{I}-2 \rho,-\rho+\mathrm{I}
$$

haben. Ich knüpfe die weitere Betrachtung an die Annahme

$$
a_{n}=2+\rho,
$$

da die übrigen fünf Fălle auf ganz entsprechende Art erledigt werden können. Ist $a_{n}=2+\rho$, so liegt $l_{n}$ im Innern der Ḱreise $(o)^{\prime}$ und $(2+o)$, wobei wieder das Komma bedeutet, dass der Rand des betreffenden Kreises mitzurechnen ist. Daher liegt $\frac{1}{k_{n-1}}=k_{n}-a_{n}$ im Innern der Kreise (o) und $(-2-\rho)^{\prime}$, folglich $k_{n-1}$ ausserhalb des Kreises (o) und im Innern oder auf dem Rande desjenigen Kreises, welcher über der Strecke - I ... als Durchmesser beschrieben ist. Da nun

$$
k_{n-1}=a_{n-1}+\frac{1}{k_{n-2}}
$$

im Innern von $\left(a_{n-1}\right)$ liegt, so muss $a_{n-1}$ einen der Werthe $-2, \rho-1,2 \rho$ besitzen. Dies ist aber unmöglich, da dann die auf $a_{n-1}$ folgende Zahl 'n nicht gleich $2+\rho$ sein könnte. Da somit die Annahme $\left|k_{n}\right|=\left|\frac{q_{n}}{q_{n-1}}\right|$ sei $\leqq$ I auf einen Widerspruch fuhrt; so gilt die Ungleichung

$$
\left|q_{n}\right|>\left|q_{n-1}\right|
$$

für jeden Werth von $n$. Aus (22) und (24) folgen nun mit Hülfe der 
oben bewiesenen allgemeinen Sätze wieder die fundamentalen Eigenschatten der hier betrachteten Kettenbruchentwicklung:

Die Entwicklung einer complexen Grösse ergiebt stets einen convergenten Kettenbruch, welcher dann und nur dann abbricht, wenn die entwickelte Grösse der Quotient zweier ganzen Zahlen $m+n \rho$ ist, und welcher periodisch wird, wenn die entwickelte Grösse einer irreductibeln quadratischen Gleichung genügt, deren Coefficienten complexe ganze Zahlen der Form $m+n \rho$ sind.

Dieser Satz kann wiederum als Fundament dienen für die Theorie der quadratischen Formen im Gebiete der complexen ganzen Zahlen, welche aus dritten Einheitswurzeln zusammengesetzt sind.

Konigsberg ${ }^{i} / \operatorname{Pr}$. den 29. November 1887. 